Développement des territoires et communication : politiques et pratiques à l'œuvre

\title{
Le développement des territoires au miroir de la communication : une problématique en perspective
}

Development of Territories in the Mirror of Communications: An Issue in

Perspective

Bruno Raoul

\section{OpenEdition}

Journals

Édition électronique

URL : http://journals.openedition.org/edc/101

DOI : 10.4000/edc.101

ISSN : 2101-0366

Éditeur

Université Lille-3

Édition imprimée

Date de publication : 1 décembre 2003

ISSN : 1270-6841

Référence électronique

Bruno Raoul, «Le développement des territoires au miroir de la communication : une problématique en perspective », Études de communication [En ligne], 26 | 2003, mis en ligne le 10 octobre 2008, consulté le 30 avril 2019. URL : http://journals.openedition.org/edc/101 ; DOI : 10.4000/edc.101

Ce document a été généré automatiquement le 30 avril 2019

(c) Tous droits réservés 


\section{Le développement des territoires au miroir de la communication : une problématique en perspective}

Development of Territories in the Mirror of Communications: An Issue in

Perspective

Bruno Raoul

1 La problématique du développement des territoires et le rapport à la communication qu'elle sous-tend, tels que l'on peut aujourd'hui les appréhender, ne peuvent l'être qu'à l'aune d'une histoire, celle de la mutation du mode d'approche de la question du territoire et, ce faisant, celle de l'évolution du mode de penser la question du développement (régional et local). C'est à construire une telle généalogie que se propose de contribuer cet article - au risque de passer par trop rapidement sur certains points - de manière à mettre en perspective la problématique en question. Ce faisant, son objet est aussi de montrer comment s'est posée la question de la communication dans son rapport au territoire et au développement (local) en en resituant le contexte, en analysant les politiques et les pratiques à l'œuvre, en faisant ressortir les enjeux, en montrant comment au cœur de la question du développement se trouve posée la question politique.

\section{Du territoire aux territoires}

\section{Le territoire à l'aune de l'aménagement du territoire (des années 50 aux années 70)}

2 Si la notion d'aménagement du territoire apparaît dans le langage officiel de l'État dès la fin de la seconde guerre mondiale, il faudra attendre une bonne dizaine d'années avant que l'on puisse parler de l'existence d'une politique dans le domaine. Entre temps, le recensement de 1954 a corroboré l'analyse effectuée par le géographe Jean-François 
Gravier, publiée sous le titre "Paris et le désert français» (Gravier, 1947) ${ }^{1}$, sur les fractures essentielles (démographiques, économiques, industrielles, sociales...) qui marquent le territoire national : en gros, les départements de l'Est et du Nord sont en plein essor industriel alors que ceux du Grand Ouest sont confrontés à un exode important (Sources : Rioux, 1983 ; Lajugie, 1985).

3 La politique d'aménagement du territoire, lancée dans un souci affiché de travailler à la cohésion économique et sociale du pays, voit véritablement le jour en 1955 à travers des dispositions réglementaires, financières et fiscales. L'année suivante sont institués des «programmes d'action régionale » sur la base d'un découpage, par l'État, du territoire national en vingt-deux " régions de programmes ", des programmes destinés à compléter le plan national. Le « fait régional » va se voir ainsi progressivement réhabilité 2 pour être finalement intégré à l'institution étatique elle-même avec la création des EPR (Établissements Publics Régionaux) en 1972.

4 L'heure est alors à la croyance dans la croissance industrielle entendue comme ferment du développement régional (et de la nation dans son ensemble), à la foi dans le progrès technique et à l'acceptation de l'urbanisation considérée comme vecteur d'émancipation (Lacour et Delamarre, 2003). Et la manière de penser la région s'en ressent. En fait, explique en 1985 le juriste J. P. Ollivaux, depuis la résurgence de l'idée régionale dans les années 50 , «le thème culturel, le droit des minorités sont délibérément évacués au profit d'une conception technocratique que l'on a appelée 'régionalisme fonctionnel' ou encore 'néo-régionalisme économique'» (Ollivaux, 1985, p. 45). Mais cette manière de penser la région n'est pas propre à l'État. On la devine dans des études réalisées par des comités régionaux, études desquelles il se dégage également une vision fonctionnaliste du développement, une vision bridée par une doctrine industrialiste3.

5 Volontarisme de l'État, planification dirigiste, technocratie, grands projets d'infrastructure, décentralisation industrielle constituent, des années 50 au début des années 70, les fondements de l'aménagement du territoire en France. Les indicateurs économiques incitent à l'optimisme. Le pays est sur la voie de la «modernisation", conduit par une autorité centrale qui se fait «Providence». Mais avec le recul, une autre perspective a percé, qui rend désuète l'ambition affichée :

«À la recherche d'une 'harmonieuse répartition des hommes et des activités sur le territoire', l'État participe en réalité à la mise en place des conditions socioéconomiques de la croissance qui s'accompagne d'une nouvelle polarisation des activités et d'une nouvelle division spatiale du travail au sein du pays d'où un renouvellement des disparités régionales" feront observer J.P. Laborie, J.P. Langumier et P. de Roo (Laborie et alii, 1985, p. 16). L'aménagement du territoire des «trente glorieuses » ne se comprend alors qu'en tant qu'il a participé du processus de taylorisation accrue de la production sur le territoire national.

6 Symptomatique de l'époque, le territoire est pensé à partir de l'État. Plus concrètement, il s'agit d'un État organisateur et modernisateur, qui détient le monopole du savoir et de l'expertise, le monopole financier et un quasi-monopole de conception et de maitrise d'ouvrage (Lacour et Delamarre, 2003, p.27). Comment alors entendre la notion de territoire?

Ce que le territoire signifie de manière générale, est, quoiqu'il paraisse, extrêmement précis : le territoire (national) relève de l'État, il entraîne et sous-tend une prédilection pour le sectoriel, une certaine négation ou une faible préoccupation pour le social (Lacour, 1985, pp. 900-901). 


\section{La montée en reconnaissance des territoires...}

7 «Comment aménager le territoire non pas pour mais avec cinquante-trois millions de citoyens ? » s'interroge Armand Frémont en 1976 (Frémont, 1976, p. 206). Dans la lignée d'une " géographie humaniste » qui commence à s'affirmer dans la première moitié des années 70 (Claval, 1996), ce géographe va proposer une théorie de l'espace vécu, basée sur la prise en compte des représentations spatiales, en considérant qu'il s'agit, dans le domaine de la géographie, « d'apprendre à penser » l'espace, ce qui ne peut être confondu avec «l'accumulation d'un 'savoir'» (ibid., p. 211) : les espaces sont vécus, imaginés, phantasmés par ceux qui y habitent (au quotidien). De ce point de vue, la "région » ne peut se réduire "à une entité strictement économique», parmi d'autres. A. Frémont, alors, n'emploie pas le terme de territoire... et pourtant. Dans la préface de la seconde édition de son livre parue en 1999, il explique :

La notion de territoire s'est substituée rapidement à celle d'espace vécu dont elle est proche sans pour autant en être synonyme. D'abord de sens commun (une portion d'espace), le territoire est devenu une entité juridique (une portion d'espace sous l'autorité d'un groupe, d'un État, d'une juridiction), puis, et de plus en plus, une notion globale, juridique, économique, sociale, culturelle, traduisant une appropriation par une collectivité sur un espace auquel elle s'identifie peu ou prou. Et de poursuivre : L'espace vécu a permis de défricher le territoire, si l'on peut dire (Frémont, 1999, p. 40).

8 Il y a ainsi évolution de la notion de territoire, d'une notion purement abstraite quand il s'agissait à travers l'aménagement du territoire d' ' aménager l'espace » à une notion plus ancrée «territorialement ", plus en prise sur les problèmes concrets et quotidiens, plus soucieuse d'identité, de social, de culture, de local. Se forge alors une autre approche du développement dans les régions :

Le social est réinventé, les potentiels locaux remis au centre de la problématique de développement, les territoires quasiment placés comme base territoriale et logique - idéologique - de l'Aménagement du Territoire. Le local devient pilote et modèle, l'État devant prendre à sa charge les grandes mutations. Se définissent alors progressivement de nouveaux rapports entre l'État et le local, entre le Territoire et les territoires. Le National reconnaît davantage l'urgence d'une autonomie accrue des acteurs locaux, comme il insiste pour une plus grande intervention des collectivités locales (Lacour, 1985, p. 926).

$9 \mathrm{Au}$ "paradigme productiviste» est opposé le «paradigme existentiel », au «développement fonctionnel» se substitue le «développement territorial», au « développement par le haut » est rétorqué le « développement par le bas ». Entérinée institutionnellement par les lois de décentralisation de 1982 qui reconnaîtront une légitimité des acteurs locaux à intervenir sur le terrain de l'aménagement et du développement, cette évolution «territoriale » se combinera avec l'affirmation, dans les régions, d'une volonté de « développement local ».

\section{... ou la revanche du développement (local)}

10 Au cours de la décennie 70, dans la lignée des idées soixante-huitardes, la contestation est à la remise en question de l'étatisme et du mode de vie industriel et urbanisé. C'est dans ce contexte bien précis qu'il faut comprendre le «retour du local ». S'opère en effet à l'époque une prise de conscience que les problèmes et les questions de société se posent 
aussi au niveau de la région, du "pays ", de la localité, du quartier. Les thèmes de l'enracinement, du quotidien, de la mémoire, de l'appartenance à un espace donné deviennent des thèmes fédérateurs. L'identité et la lutte ne sont plus envisagées sous l'angle exclusif d'un rapport de classes et de l'exploitation dans la production (Mattelart, 1986, p. 176). Un slogan devient emblématique : «Vivre et travailler au pays » (Dubet, 1983). Le « local » est vu comme nouvel « espace » à investir socialement, culturellement, politiquement pour repenser la démocratie et repenser la société d'une façon plus générale, il est vu comme "lieu» à partir duquel on pourra changer la vie. Un des intervenants au colloque consacré à «l'objet local » (tenu en 1975 et dirigé par Lucien Sfez) y discerne la concrétisation d'« une avancée de la conscience sociale » (Sfez, 1977, p. 295). Ainsi, la notion de « local » ne peut-elle être circonscrite au seul sens de "localité », de «ville», de "pays». Ce n'est pas seulement ni avant tout de géographie qu'il s'agit alors, l'objet en question est fondamentalement d'ordre politique.

11 Très tôt, la question du local est aussi pensée de manière communicationnelle, les technologies en essor à l'époque (radios "locales», vidéo «militante», télévision « de proximité ») et d'autres supports comme les journaux associatifs et journaux de quartier étant vus comme moyens possibles pour dynamiser la communication sociale au niveau local (Simon, 1995) mais aussi pour activer, rendre visible et gérer des luttes urbaines, voire les mettre en mémoire (Duguet, 1981).

Le concept de développement subit alors une évolution sémantique sous la pression de ces mouvements sociaux se réclamant du «droit à la différence » et de la " participation » pour dénoter des revendications sur « la qualité de la vie » et faire valoir une aspiration à « l'autonomie », à « l'alternative autogestionnaire ». «La notion de développement local apparait comme la symbolisation du long cheminement critique effectué par ceux qui se sont opposés au modèle dominant de développement » relève ainsi le sociologue Roger Benjamin (Benjamin, 1984, p. 17).

13 Vecteur d'une nouvelle philosophie du développement soucieuse des histoires et des cultures des "individualités micro-régionales", modèle alternatif qui intégrerait la dimension économique dans le respect de la multiplicité et de la diversité des territoires, le développement local, en prenant appui sur le potentiel endogène, entend privilégier l'idée de communauté d'intérêt de l'espace local - au risque toutefois de construire la fiction d'une unicité d'intérêts entre les différents acteurs locaux. Au début des années 80 , certains y discernent un mouvement « en train de passer du stade du militantisme à celui de l'élaboration conceptuelle » (Chassagne, 1983, p. 31), sachant que « dans le retour au local, il y a aussi l'espoir de dépasser la crise » (Mathieu, 1983, p. 28).

Une dizaine d'années plus tard, le débat théorique bat son plein chez les chercheurs en économie régionale et en géographie économique entre ceux qui modélisent la réussite des «régions qui gagnent » et ceux qui pointent l'intégration du local à une logique capitaliste globale d'une part et l'accroissement des inégalités spatiales d'autre part (Benko et Lipietz, 1992). Devant le constat de pratiques de développement local (dans le domaine industriel en l'occurrence) qui, à l'encontre du modèle fordiste poussant à la normalisation et à l'uniformisation, "valorisent la richesse des différences comme facteur de développement ", un économiste qui sera partie prenante du débat se risque à affirmer : « Le développement local n'est pas une idéologie en ce sens qu'il ne trace pas de chemin prospectif linéaire » (Pecqueur, 1989, p. 146). 
Or, dans l'intervalle, le sens et la portée du «développement local», dans sa version dominante - «La dimension industrielle de résistance puis de réorganisation face à la crise l'emporte largement sur l'ancienne conception à fondement rural du 'développement des Pays' » notent ainsi en 1990 Robert Boure et Jean Menville (Boure et Menville, 1990) -, se sont modifiés, jusqu'à engendrer une véritable rupture conceptuelle. Le développement local s'est défait peu à peu de ses aspirations démocratiques originelles et de ses prétentions à asseoir un nouveau projet de société pour se rallier aux impératifs de l'économisme ambiant et intégrer les normes de gestion de la compétition concurrentielle, pour se métamorphoser en doctrine économique pour le local. Au seuil des années 90 , le développement local se révélera bien plus une modalité d'ancrage territorial de l'hégémonie capitaliste qu'il ne dévoilera un nouveau mode de développement (Raoul, 1994). Dans le même mouvement, la communication, dans son rapport au local, dérivera d'une conception assise sur l'idéal de la participation et de la citoyenneté à une conception instrumentale et gestionnaire.

\section{Les territoires investis par la communication : l'emprise d'une conception techniciste et managériale}

16 Les années 80 seront marquées par l'arrivée sur le terrain du local d'une nouvelle catégorie d'acteurs socioprofessionnels, forts de compétences dans la dynamisation économique des territoires. Dans les collectivités locales, la légitimité de l'action n'est plus alors fondée sur la primauté du droit mais sur «l'efficacité économique » explique Alain Faure, les maires se devant d' incarner une certaine symbolique gestionnaire » (Faure, 1991, p. 125). L'heure est donc, pour les édiles locaux, au recours aux méthodes managériales et entrepreneuriales pour relancer le développement territorial (Padioleau, 1991).

Dans cet élan de professionnalisation, le développement local se voit investi, entre autres, par des cabinets d'audit et de conseil en communication dont l'action vise à forger une certaine image du local : modernité, vitalité, recherche, haute technologie, agréments... Les villes et les régions se rallient à la cause du marketing. Leur image est mise à l'affiche, leur territoire s'offre aux investisseurs. Mais l'enjeu, c'est aussi, étant donné les compétences nouvelles reconnues aux collectivités locales, la reconnaissance de la légitimité de celles-ci par le public local. Il s'agit de « vendre » aux administrés des projets d'aménagement et d'équipement, tout au moins de les faire adhérer non à une politique mais à des événements, au risque que la communication municipale ne se dilue dans la communication politique (Miège, 1989; Pailliart, 1993a). Le citoyen-administré se voit réduit à un statut de consommateur d'images du local. Du côté des économistes régionaux, certains iront jusqu'à dénoncer «la dictature des spécialistes de la communication »:

Il faut vendre son site, et personne n'hésite, sans voir que cela confine au ridicule, à présenter sa commune, son canton ou sa région comme étant, au vu d'une carte, au centre du monde. La perversité de la fièvre communicationnelle est de présenter un territoire comme un formidable lieu de synergie sans jamais se préoccuper de vérifier si cela est vrai auprès des acteurs concernés. Il s'agit dans la plupart des cas d'une politique de communication visant à pallier artificiellement les carences de communication entre acteurs (Pecqueur, 1991). 

d'émergence de nouvelles technologies (câble, télématique), auréolées d'une soi-disant capacité à susciter la décentralisation d'activités de production et de service d'une part et la participation de tout un chacun à la gestion de la cité d'autre part, vient corroborer l'idée que le salut du développement local est aussi dans la communication. Dans la seconde moitié des années 80 , le rapport de certaines villes aux TIC franchit une nouvelle étape avec les projets de téléports. L'objectif est, en s'affranchissant du monopole de l'opérateur public, de proposer en propre des conditions d'accueil particulièrement intéressantes en matière de télécommunication et ainsi conforter un pouvoir d'attraction des entreprises, notamment les étrangères, dans un cadre dorénavant concurrentiel entre collectivités locales. Outre de témoigner que la pression dérégulatoire s'est aussi exercée par le bas, ces projets sont révélateurs de l'évolution, au cours de la décennie, des modes de légitimation des actions des responsables locaux en matière de TIC qui passent d'une perspective politique et socio-culturelle à une perspective économique et gestionnaire, d'une perspective de communication sociale à une perspective de communication instrumentale. Constat semblable a été fait à propos des services télématiques municipaux (Pailliart, 1993b).

19 À travers une telle réflexion sur le rapport de la communication au local, nous avons pu apprécier comment, de manière différenciée dans le temps, le concept de communication a donné sens à «l'objet local » et donc saisir comment, au cours de la décennie 80, le discours et la pratique du développement local se sont greffés sur l'évolution paradigmatique de l'idée même de communication (technicisation, professionnalisation, déréglementation, recours au marketing et à la publicité) pour se défaire peu à peu de leurs références originelles. En d'autres termes, nous avons assisté durant cette période à un processus d'aseptisation politique tant de la question du développement local que de celle de la communication locale.

\section{Une nouvelle culture de politique publique dans les années 90}

\section{Le développement des territoires à l'heure du « contrat » et du « projet »}

Dans la lignée des nouvelles modalités relationnelles entre l'État et les collectivités territoriales établies par les lois de décentralisation de 1982 (à travers notamment les Contrats de Plan État-Région), se conforte dans les années 90 une nouvelle culture de politique publique par laquelle est repensé le mode de positionnement des différents acteurs impliqués dans l'aménagement et le développement territorial.

Après un grand relâchement de la part de l'État dans la deuxième moitié des années 80 (les idées libérales et déréglementaires ont de plus en plus le vent en poupe), l'aménagement $\mathrm{du}$ territoire va de nouveau constituer une préoccupation gouvernementale à la fin de la décennie et se voir totalement réhabilité au cours des années 90 par des gouvernements de couleurs politiques opposées. Les textes adoptés en 1995 puis en 1999, associant explicitement aménagement et développement, se remarquent d'autant plus qu'ils réservent une place toute particulière au "pays ", une notion quelque peu tombée en désuétude depuis la décentralisation. Ceci dit, le "pays » 
s'entend, signe des temps, comme « territoire de projet ». En effet, il ne s'agit plus tant de viser à une meilleure répartition spatiale des hommes et des activités que de favoriser, par un cadrage, une incitation et un soutien étatiques, un développement local et régional dynamisé par les acteurs locaux eux-mêmes (cf. infra). Autre signe des temps, la « recomposition des territoires » est envisagée à l'aune du développement durable.

Mais le thème du développement des territoires dans les années 90, c'est aussi le renforcement de la politique de la ville. Amorcée dans les années 80 devant une situation urbaine de plus en plus sensible dans certains quartiers (augmentation du chômage, dégradation de l'habitat et concentration de pauvreté dans les grands ensembles et dans des villes en déclin industriel...), elle va de plus en plus prendre la forme d'une préoccupation $\mathrm{du}$ politique pour la question du développement social et socioéconomique (logement social, éducation, santé, culture, revitalisation économique des quartiers en difficulté, sécurité, déplacements), à côté de la question du bâti et de l'aménagement. Une tout autre conception du développement local que celle qui, marquée par l'économisme (et l'industrialisme) ambiant, a dominé au cours des années 80 , s'affirme alors. Elle contribuera à l'arrivée, dans les villes, de nouveaux professionnels et de nouvelles compétences contribuant à faire avancer la réflexion des collectivités territoriales (et des élus) sur le développement social urbain et à dynamiser l'action locale (Peillon, 2001). Avec la politique de la ville, il y va d'une prise de conscience que la problématique du développement local est aussi au cœur de l'urbain. Formulé en des termes plus circonstanciés, cela donne : «Comment transformer en véritables morceaux de ville, en dynamisme permanent, des territoires où l'État-providence s'en est tenu trop longtemps à une assistance qui a mal pris en considération toute la richesse humaine des potentialités locales?", alors même que la politique en question peut être en tension avec un autre objectif, en fait " majeur ", celui de « la paix sociale » (Chaline, 2003, p. 68).

En termes de tendances de fond, les politiques publiques des années 90 concernant le territoire se traduisent en définitive par un encouragement à l'intercommunalité ou à une approche intercommunale des questions (de là une nouvelle manière de penser l'échelle institutionnelle locale), par l'importance d'envisager la question du développement simultanément à celle de l'aménagement et par une nouvelle culture politique basée sur le contrat et le projet, ce qui induit de nouvelles procédures et de nouvelles pratiques impliquant de la part des acteurs locaux et régionaux une prise en charge et une responsabilité accrues tant dans l'élaboration que dans la mise en œuvre de la politique. L'approche institutionnelle du territoire sous-tend donc l'idée que le territoire recouvre différentes catégories d'acteurs (personnes, groupes de personnes, associations, institutions locales) qui ont une légitimité et une capacité à agir dans l'espace donné. Il ne s'agit donc plus d'une conception étroitement spatiale d'un espace à aménager, au risque toutefois de laisser filer le glissement sémantique et de ne prendre garde à l'écueil sociologiste quand la notion renvoie purement et simplement aux seuls acteurs (locaux). Une tension n'en est pas moins toujours sensible entre une conception fonctionnelle et une conception « territoriale » du territoire quand, par exemple, celui-ci est envisagé comme espace de mise en œuvre d'un programme de diffusion des TIC dans une région (Raoul, 2002). 


\section{Penser la communication dans les territoires}

24 beaucoup évolué ces dernières années. Après une période d'euphorie pour la libéralisation du marché des télécommunications, l'acteur politique fait de plus en plus retour dans le secteur, notamment au nom de l'aménagement du territoire. Depuis peu, les collectivités territoriales sont autorisées à investir dans les réseaux, et pourraient se voir reconnaître le droit d'«exercer des fonctions d'opérateur» si la proposition formulée par le gouvernement lors du comité interministériel d'aménagement du territoire de décembre 2002 débouche sur une modification de la loi. On voit mieux ici en quoi, dans ce domaine, la déréglementation s'est conjuguée avec la décentralisation.

Ceci dit, on le sait, les mythes ont la vie dure. Mais on a beau le savoir, la relâche les concernant tiendrait du renoncement à l'exercice de la pensée critique. "Les technologies de la communication permettent de s'affranchir des contraintes géographiques » peut-on lire en sous-titre d'un article dont le titre en lui-même est tout à fait au diapason de l'euphorie technologiste du moment : «La nouvelle économie donne leur chance à tous les territoires » (Le Monde, 23 juin 2000). Dans un petit guide pratique réalisé par la DATAR à l'intention des "acteurs de la recomposition des territoires ", il n'est pas surprenant d'y déceler un ton préventif : «Le déploiement sur l'ensemble du territoire de réseaux à haut débit est identifié comme la clef du développement local et comme un enjeu décisif pour la décennie à venir en matière d'aménagement du territoire » (Cassette, 2002, p. 20). Mais, indépendamment du côté lobbyiste qui se dégage de l'ouvrage, la formule ici reprise a tout de la «métaphore mécanique " (Drewe et Joignaux, 2002), quand bien même nombre de travaux de recherche ont montré qu'en matière de rapport réseaux/territoires, "c'est la pénurie qui structure » (Musso et Rallet, 1995 ; Lefebvre et Tremblay 1998 ; Musso, 2002).

D'autre part, le réseau internet a redonné du crédit à la thèse comme quoi les TIC pourraient être au fondement d'une redynamisation du débat démocratique et de la participation. À ce sujet, une réflexion d'Isabelle Pailliart permet de faire le lien avec le contexte dans lequel se déploie de nouveau, au niveau local, cette question des TIC :

Les possibilités d'interactivité technique mettent en évidence la figure d'un habitant actif, susceptible de s'impliquer dans les affaires locales tout comme les opérations de concertation jouent aussi sur le registre de l'implication de l'habitant, de sa transformation en citoyen actif et responsable (Pailliart, 2000).

En effet, à la charnière des deux siècles, on a assisté à un retour en force, dans le discours politique et dans les décisions législatives, de la question de la participation des habitants (instauration des conseils de développement pour les pays et les agglomérations, concertation obligatoire pour les plans locaux d'urbanisme, implication des habitants dans les contrats de ville, création des conseils de quartier...). Est-ce là le signe d'une repolitisation du débat autour de la communication locale?

Le principal changement, explique Loïc Blondiaux (IEP Lille) à propos de la « démocratie participative »- tout en relevant la « vrai pauvreté de la réflexion » sur ce thème -, tient dans le fait qu'il s'agit d'une révolution dans la manière de penser la politique: "Aujourd'hui, c'est la manière dont on arrive à la décision qui compte plus que celui qui décide $»^{3}$. Autre changement pointé : la " démocratie participative » a été changée, dans le texte de loi adopté en 2002, en «démocratie de proximité ». Pointer cela, c'est une 
manière de faire ressortir que, ce faisant, c'est le « local » qui se trouve réhabilité et qu'il est confirmé, selon le mot de $\mathrm{D}$. Wolton, «comme la première étape d'une revitalisation de la politique », au risque, précise ce dernier, d'une survalorisation théorique et politique si ne lui est pas retiré « ce parfum de pureté originelle » (Wolton, 2000, p. 89). La communication dans son rapport à la démocratie au niveau local n'est donc plus envisagée, contrairement à une tendance assez forte lors de la montée en force d'internet, sous l'angle principal de l'interactivité technique.

Sans oublier cependant la persistance dans les territoires du modèle publicitaire et marketing de la communication, l'enjeu de celle-ci s'apprécie aussi, outre en termes de coordination de différentes catégories d'acteurs ayant des façons différentes de penser le territoire et leur insertion dans le territoire, à l'aune d'une capacité à l'appropriation sociale et collective des moyens d'expression et de communication. Dès lors, au-delà du droit à l'expression, est-ce en termes non seulement de maitrise technique des outils qu'il convient de raisonner mais également - et surtout - en termes de rapport à l'écriture, à l'image et à l'expression orale et en termes de capacité à s'inscrire dans l'espace de la communication locale. En ce sens, la démocratie participative - si l'on veut bien entendre qu'elle englobe dans un sens large l'idée qu'un collectif de citoyens, légitimement réunis, se sente apte à se saisir d'une question d'ordre social, politique, culturel, économique ou autre, en toute autonomie, indépendamment de l'agenda institutionnel du pouvoir municipal, pour l'introduire dans l'espace du débat politique local - n'est pas avant tout une question d'ordre juridique et institutionnel. Elle est principalement de l'ordre du culturel : dans quelle mesure les cadres cognitifs et normatifs par lesquels est pensée et régulée la vie sociale aujourd'hui amènent-ils les citoyens à "se projeter" comme pouvant s'impliquer, à se sentir acteurs du fonctionnement et de la régulation de la société au quotidien et à plus long terme ? Politiquement parlant, cela ne va pas de soi, la participation relevant, pour reprendre le mot de L. Quéré, d'un "dispositif historicoculturel de symbolisation ou d'objectivation sociale » (Quéré, 1982, p. 157). Les militants du local et de l'éducation populaire des années 70 et du début des années 80 ne manqueront pas de rappeler qu'il n'y a rien de fondamentalement nouveau à le dire. A tout le moins reconnaîtront-ils que ce sont des questions qui semblent faire retour après un assez long silence, un retour qui s'inscrit aujourd'hui sur un nouveau fond politique, la " participation » étant actée institutionnellement.

Il n'empêche, ce sont là des domaines investis par différentes disciplines scientifiques, entre autres les sciences de l'information et de la communication. Et ce sont là des questions à investir plus encore. Il y va de la compréhension, à l'échelle locale, des modalités de redéfinition de l'espace public et de recomposition des territoires aujourd'hui, de la compréhension de la question identitaire et de la question politique.

\section{BIBLIOGRAPHIE}

Alvergne, C. et Musso, P., (2003), Les grands textes de l'aménagement du territoire et de la décentralisation, Paris, La Documentation française/DATAR. 
Benjamin, R., (1984), « Développement local : la notion de développement a-t-elle encore un sens? », Recherche sociale, $n^{\circ} 89$, janvier-mars 1984, pp. 12-26.

Benko, G. et Lipietz, A., (sous la dir.), (1992), Les régions qui gagnent. Districts et réseaux : les nouveaux paradigmes de la géographie économique, Paris, P.U.F.

Boure, R. et Menville, J., (2002), « Sur et sous le 'local' », Les Cahiers du Lerass, n²0, mai 1990, pp. 7-18.

Cassette, B., (2002), Le développement numérique des territoires, Paris, La Documentation française/DATAR, Coll. « Territoires en mouvement ».

Chaline, C., (2003), Les politiques de la ville, Paris, P.U.F., Coll. « Que sais-je ?», n³232.

Chassagne, M. E., (1983), « Du refus de la mort à l'auto-création », Autrement, « Le local dans tous ses États », n47, pp. 29-35.

Claval, P., (1996), Histoire de la géographie, Paris, P.U.F., Coll. « Que sais-je ?», nº65.

Drewe, P. et Joignaux, G., (2002), « Réseaux et territoires : retour vers quelques mythes », in : Musso P., Le territoire aménagé par les réseaux, Paris, Éditions de l'Aube/DATAR, pp. 35-54.

Dubet, F., (1983), « Des nations sans État aux pays de l'État », Autrement, « Le local dans tous ses États ", n47, pp. 10-15.

Duguet, A. M., (1981), Vidéo, la mémoire au poing, Paris, Hachette, Coll. « L'Échappée belle ».

Faure, A., (1991), « Pouvoir local en France : le management mayoral à l'assaut du clientélisme », Politiques et management public, vol. 9, $\mathrm{n}^{\circ 4}$, septembre 1991, pp. 115-132.

Fremont, A., (1976 et 1999), La région, espace vécu, Paris, P.U.F., coll. « Le géographe » (Deuxième édition en 1999 : Champs-Flammarion).

Gravier, J. F., (1947), Paris et le désert français, Paris, Le Portulan.

Laborie, J. P., Langumier, J. P. et Roo, P. de, (1985), La politique française d'aménagement du territoire de 1950 à 1985, Paris, La Documentation française.

Lacour, C., (1985), «Les méthodes d'analyse de l'espace », in : Lajugie J., Delfaud P. et Lacour C., Espace régional et aménagement du territoire, Paris, Dalloz, 2e édition, 987 p., 4e Partie, pp. 649-930.

Lacour, C. et Delamarre, A., (2003), 40 ans d'aménagement du territoire, Paris, La Documentation française/DATAR, Coll. « Territoires en mouvement ».

Lajugie, J., (1985), « L'élaboration de la théorie économique spatiale et l'apparition de la politique d'aménagement du territoire et de développement régional », in : Lajugie J., Delfaud P. et Lacour C., Espace régional et aménagement du territoire, Paris, Dalloz, 2e édition, 987 p., 1er Partie, pp. 5-166.

Lefebvre, A. et Tremblay, G., (1998), Autoroutes de l'information et dynamiques territoriales, SainteFoy (Québec) - Toulouse, Presses de l'Université du Québec - Presses Universitaires du Mirail.

Mathieu, N., (1983), « Les riches heures de la notion de pays », Autrement, n47, pp. 23-28.

Mattelart, A. et Mattelart, M., (1986), Penser les médias, Paris, La Découverte.

Miege, B., (1989), La société conquise par la communication, Grenoble, PUG.

Musso, P. et Rallet, A., (sous la dir.), (1995), Stratégies de communication et territoires, Paris, L'Harmattan. 
Musso, P., (2002), Le territoire aménagé par les réseaux, La Tour d'Aigues, Éditions de l'Aube/DATAR.

Ollivaux, J. P., (1985), La décentralisation. La région et l'aménagement du territoire, Paris, Syros-Adels.

Padioleau, J. G., (1991), « L'action publique urbaine moderniste », Politiques et management public, vol. 9, n³, septembre 1991, pp. 133-143.

Pailliart, I., (1993a), Les territoires de la communication, Grenoble, PUG.

Pailliart, I., (1993b), « Les services télématiques municipaux : la médiation impossible ? », Les Papiers, $n^{\circ} 11$, Presses de l’Université du Mirail.

Pailliart, I., (2000), « Les enjeux locaux de la démocratie électronique », Hermès, n²6-27, CNRS Éditions, pp. 129-138.

Pecqueur, B., (1989), Le développement local, Paris, Syros-Alternatives.

Pecqueur, B., (1991), « L'avenir du local est-il dans le partenariat? », Territoires, n³23, décembre 1991.

Peillon, P., (2001), Utopie et désordre urbains. Essai sur les grands ensembles d'habitation, La Tour d'Aigues, Éditions de l'Aube (Chap. 6 : « Un quart de siècle de politique de réparation », pp. 265-277).

Phlipponneau, M., (1956), Inventaire des possibilités d'implantations industrielles en Bretagne, CELIB.

Quéré, L., (1982), Des Miroirs Équivoques. Aux origines de la communication moderne, Paris, AubierMontaigne.

Raoul, B., (1994), « Communication, territoire, mutations industrielles : l'industrialisation régionale entre développement local et globalisation ", Thèse de doctorat (nouveau régime) en sciences de l'information et de la communication (sous la direction d'A. Mattelart), Rennes, Université de Rennes II - Haute-Bretagne.

Raoul, B., (2002), Politique publique, médiation sociale, territoire : le cas de « l'opération Cyber Nord/Pas-de-Calais », Rapport de recherche (avec la collaboration de Stéphane Benassi pour l'enquête de terrain), Lille, IFRESI - GERICO.

Rioux, J. P., (1983), La France de la IVe République. 2. L'expansion et l'impuissance. 1952-1958, Paris, Seuil, collection Points, 2e Partie, chap. 5, « L'aiguillon de la croissance », pp. 167-211.

Sfez, L., (sous la direction de), (1977), L'objet local, Paris, UGE, 10/18.

Simon, J. P., (1995), « Petite histoire des politiques de communication locale de 1970 à nos jours ", in : Musso P. et Rallet A. (sous la dir.), Stratégies de communication et territoires, Paris, L'Harmattan, pp. 125-151.

Wolton, D., (2000), « Le local, la petite madeleine de la démocratie », Hermès, $n^{\circ} 26-27$, CNRS Éditions, pp. 89-97.

\section{NOTES}

1. Pour une approche critique de ce livre, voir Alvergne et Musso, 2003, et Lacour et Delamarre, 2003.

2. Tenue en suspicion après la Révolution française car associé aux provinces de l'Ancien Régime (Ollivaux, 1985), l'idée de région a été discréditée au sortir de la guerre 39-45 non seulement en raison de l'instauration par le régime de Vichy de préfectures régionales, mais également du fait de la « collaboration » de certains courants régionalistes - notamment en Bretagne. 
3. "Redonner sens à la démocratie participative ", intervention au Jeudi de la Ville (journée d'information, de réflexion et de débat organisée par l'IREV Nord/Pas-de-Calais - Institut régional de la Ville), 27 mars 2003.

4. Ainsi en Bretagne. Le recensement de 1954 l'a montré, l'exode est le nœud gordien du « problème breton ». Aussi la revendication du CELIB, le Comité d'études et de liaison des intérêts bretons, est-elle limpide sous la plume du géographe Michel Phlipponneau, théoricien de la « géographie appliquée » et membre fondateur du comité, dans l'« inventaire des possibilités d'implantations industrielles en Bretagne », paru en 1956 : «Seul un développement rapide de l'industrie peut résoudre le problème breton ». Et le géographe d'argumenter, d'une manière pour le moins condescendante, dans le sens de l'intérêt de décentraliser des industries vers la Bretagne : outre des raisons administratives (le régime de l'agrément), ou techniques et financières, " l'avantage essentiel est constitué par la présence d'une main d'œuvre calme, consciencieuse, s'adaptant remarquablement au travail à la chaîne des usines modernes et dont les salaires réels demeurent nettement plus bas que ceux des ouvriers parisiens » (Phlipponneau, 1956, p. 13).

\section{RÉSUMÉS}

Cet article propose une mise en perspective généalogique de la problématique du dossier tout en voulant pointer, sur la fin, certains des questionnements qui la travaillent aujourd'hui. Dans un premier temps, l'auteur montre que si, dans les années 50 et 60 , le territoire s'est entendu à l'aune de l'État et du niveau national, une autre conception, plus soucieuse du social et du local, s'est affirmée dans les années 70 jusqu'à soutenir une nouvelle approche du développement territorial. Puis il explique qu'au cours des années 80, le développement local est progressivement passé du stade du militantisme à celui de modèle de développement économique pour le local, un mouvement au cours duquel la communication, dans son rapport au local, a dérivé d'une perspective participative et citoyenne à une conception instrumentale et gestionnaire. Après une décennie marquée par l'émergence d'une nouvelle culture de politiques publiques, le début des années 2000 annonce-t-il une réhabilitation du politique au niveau local ? Dans la réflexion finale, l'article s'attache à indiquer quelques conditions d'une telle réhabilitation.

This paper proposes a genealogical approach to the problematics of the document, focusing on some of the questions that structure it today. It first shows that while in the 1950s and the 1960s, the territory was understood from the standpoint of state and at national level, another conception, more concerned with the social and the local, asserted itself during the 1970s until it entailed a new approach of territorial development. It then explains how during the 1980s, local development gradually passed from the activist stage to a model of local economic development. In the process, communication in its relationship with the local drifted from a participatory and citizen-related perspective to an instrumental and management-oriented conception. Following a decade marked by the emergence of a new public policy culture, does the beginning of the 2000 s forecast a restoration of politics at the local level? In a final reflection, the paper pinpoints some of the conditions for such a restoration. 
INDEX

Keywords : territorial development, political communication, country planning, territorial management, ICT

Mots-clés : développement territorial, communication politique, aménagement du territoire, management territorial, TIC

\section{AUTEUR}

\section{BRUNO RAOUL}

Gerico - Université Charles-De-Gaulle - Lille 3 